\title{
A Method of Inspection Simulation Analysis Based on VERICUT in Three-Dimensional Inspection Planning
}

\author{
G.J. Duan, J.A. Zhao \\ School of Mechanical Engineering and Automation \\ Beihang Univ. \\ Beijing, China
}

\begin{abstract}
Aiming at rising the efficiency and accuracy in inspection planning and avoiding interferences in actual inspection, a method of inspection simulation analysis based on VERICUT, a simulation software based on $\mathrm{CNC}$ (Computer numerical control) in three-dimensional inspection planning was proposed. In this paper, data processing of the measure points array, the generation of the CMM(Coordinate Measuring Machining) program and the simulation analysis on the platform of VERICUT were discussed. And a virtual CMM system was established.
\end{abstract}

Keywords-Simulation Analysis; VERICUT; CMM; Inspection Planning

\section{INTRODUCTION}

With the rapid development of Computer Integrated Manufacturing System and Computer Aided Process Planning, Computer Aided Inspection Planning (CAIP) was promoted remarkably as a bridge between manufacture and inspection. Simulation analysis is one of the most important segments in three-dimensional inspection planning. It is also an important part of the CAIP system[1].

After measurement points planning and route planning, a measurement points array and a position points array were generated attached to the three-dimensional model. All of the information was recorded in the file of CAIP system named IP $\mathrm{Xml}$. The file was according to the rule of $\mathrm{Xml}$, and by resolving this file, a simulation points array were created. Furthermore, the CMM program could be generated. By loading the program and importing the models of virtual CMM in VERICUT, the environment of inspection could be accomplished. After building the probe library, setting the basic parameters of the virtual CMM and loading the part to be inspected, the simulation could be running. After the simulation, Collision positions and the corresponding codes would be shown in the window of VERICUT, which make it possible for the inspectors to modify the CMM program.

Under normal circumstances, all of the work mentioned above was executed on real CMM machine. By finishing all these processes via the virtual CMM, the efficiency of inspection is promoted and the safety level of the CMM machine is updated.

Scholars have launched an in-depth study on simulation analysis in three-dimensional inspection planning. There are two kinds of ways to accomplish the simulation. The most common way is based on OpenGL. Virtual CMM system was set up by calling functions from OpenGL. The other way is based on the CNC machining part of the CAD software, which is limited and short in versatility. According to the studies on simulation analysis, the author attempts to do the simulation analysis by using VERICUT[2-5].

VERICUT is customized for CNC simulation, which is good at interference check. It also contains interfaces to varieties of CAD softwares such as CATIA, Pro/E, Solid works, etc. That is why VERICUT is chosen to applied for simulation analysis in three-dimensional inspection planning.

\section{System Design Scheme}

Simulation program is a connection between the CAIP file and the VERICUT. There are three parts in generating the simulation program, the foreground, the background and the statistic layer. By choosing the exact $\mathrm{Xml}$ and path, analysis of the corresponding points' array would be done in the background and the data would be saved logically. Meanwhile, the swing angle of each measurement point was calculated and saved. Then, the safety height should be given, and it would also be saved in the statistic layer. When setting the name and path of the program, the calculation of the simulation path would be done in the background and the simulation points array would be generated. Eventually, the CMM program was created and saved in the statistic layer.

VERICUT is the terminal of the simulation system. Initially, the virtual CMM system should be set, which is the basic environment of the simulation. Then, the probe library should be well-designed to ensure the precision of the simulation. After loading the part to be inspected and the program, the simulation of inspection could be started. The system design scheme is shown in figure 1 .

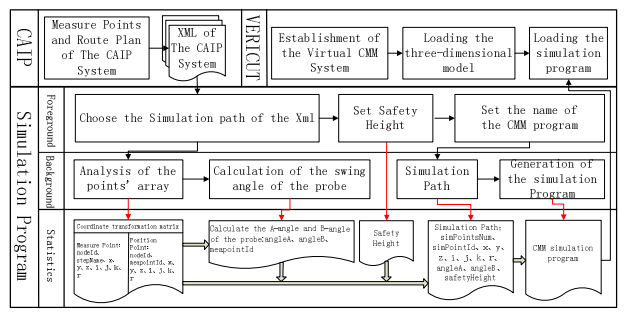

FIGURE I. .SYSTEM DESIGN SCHEME. 


\section{VIRTUAL CMM SYSTEM}

\section{A. Control System}

The Virtual CMM System consists of Control System and the Entity of the CMM. And the Control System is the core of the simulation.

The major function of the Control System is to analyse CNC codes, including $\mathrm{G}$ codes, $\mathrm{M}$ codes, etc.

Some of the codes could be used directly for inspection simulation, such as "G00" for moving the virtual CMM to the exact point swiftly. However, traditional $G$ codes could not meet the requirements of some inspection features such as holes. To inspect the feature such as a hole, the coordinates and the vector of the hole-centre and the radium of the hole would be given, and by analysing these statistics, the CMM could accomplish the measurement of a hole. So a development of the Control System is necessary and important.

\section{B. Entity of CMM}

The modelling accuracy of the virtual CMM's entity determines the precision of the simulation. There are two ways to modelling the entity. One is mapping and the other one is by parametric modelling of the Pro/E.

Build three-dimensional simulation model of the CMM entity by CAD software such as Pro/E or CATIA. Export the models in the format of .stl. Create a new project of virtual CMM in VERICUT and import the .stl models. Add five axis $\mathrm{X}, \mathrm{Y}, \mathrm{Z}, \mathrm{A}, \mathrm{C}$ in the new virtual CMM. Associate the models with the axis and set the motion modes of the axis. Establish the coordinate system of the virtual CMM and set the collision detection, travel limits and the initial position of the CMM. The process is shown in Figure 2.

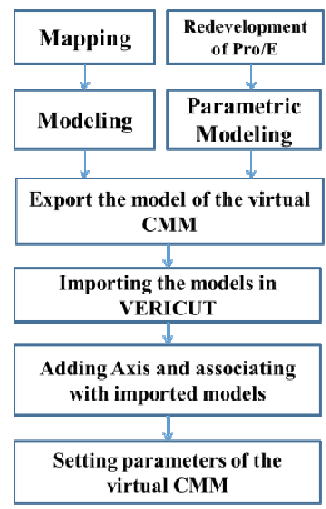

FIGURE II. PROCESS OF CMM'S ENTITY BUILDING.

\section{Probe Library}

The establishment of the probe library is based on the tool library of VERICUT. The file of the tool library is according to the rule of Xml which could be revised and edited. All of the information including the length, diameter, clamping points of the probe was recorded in this file. By resolving the file, the parameters of the probe could be defined and new probes could be built.

The probe library could be visited by the inspectors via the interfaces. The whole process is shown in figure 3 .

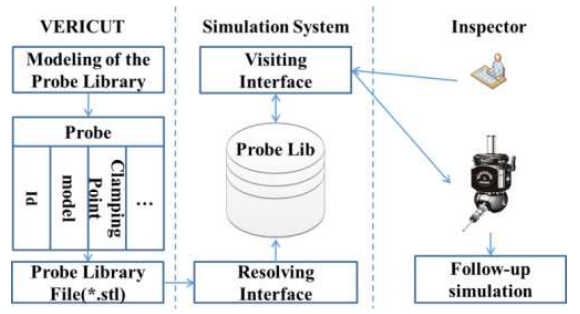

FIGURE III. PROCESS OF VISITING PROBE LIBRARY.

\section{Generation OF the Simulation ProgRAm}

A. Swing Angle

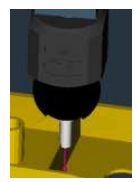

(a)

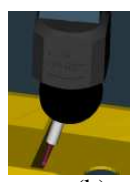

(b)

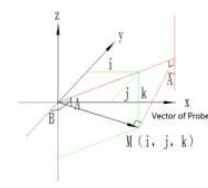

(c)
FIGURE IV. SWING ANGLE COMPUTATION.

In order to avoid the interference to the most extent, a series of rational swing angles would be computed. As is shown in Figure4, the swing angle in (b) is much more proper than the one in (a).

In figure $4(\mathrm{c}), \mathrm{M}(\mathrm{i}, \mathrm{j}, \mathrm{k})$ is the vector of the measurement point and the vector of probe should be perpendicular to $M$. The angle between the vector of probe and Z-axis negative is defined as angle $\mathrm{A}$, and the angle between the vector of probe in the projection of the $\mathrm{XY}$ plane and $\mathrm{Y}$-axis negative is defined as angle $\mathrm{B}$.

The calculation of angle A and angle B could be described as eqn (1) and eqn (2).

$$
\begin{gathered}
A=\left[\left(\operatorname{acos}(k) \cdot \frac{180}{\pi}-90\right) \mid 15\right] \cdot 15(1) \\
B=\left[\left( \pm 90+\operatorname{atan}\left(\frac{j}{i}\right) \cdot \frac{180}{\pi}\right) \mid 15\right] \cdot 15(2)
\end{gathered}
$$

\section{B. Points Array}

According to the measurement points and position points obtained from the $\mathrm{Xml}$, arrange the points in a certain order to form the simulation points array.

If the path contains only one feature to inspect, one measurement point has only one position point to match. But if there are more than one feature to inspect, more position points are required as the safety position points so as to avoid collision when crossing the features.

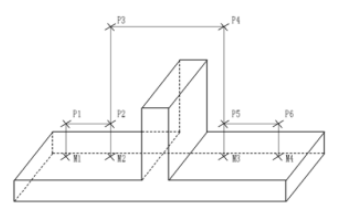

FIGURE V. EXAMPLE OF POINTS ARRAY.

In figure $5, \mathrm{M}_{\mathrm{i}}$ are the measurement points and $\mathrm{P}_{\mathrm{i}}$ are the position points, the simulation points array are: 
$\mathrm{P} 1 \rightarrow \mathrm{M} 1 \rightarrow \mathrm{P} 1 \rightarrow \mathrm{P} 2 \rightarrow \mathrm{M} 2 \rightarrow \mathrm{P} 2 \rightarrow \mathrm{P} 3 \rightarrow \mathrm{P} 4 \rightarrow \mathrm{P} 5 \rightarrow$

$\mathrm{M} 3 \rightarrow \mathrm{P} 5 \rightarrow \mathrm{P} 6 \rightarrow \mathrm{M} 4$

\section{Simulation Program}

The simulation program is formed based on the simulation points array and the format that the control system could be resolved. Two kinds of programs are formed. One is the main program and the other is subprogram. Main program is generated according to the simulation points array. And subprogram is generated based on the specific feature such as a hole and the control system. 6.

The process of simulation programming is shown in Figure

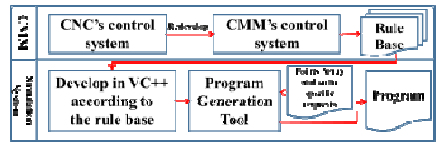

FIGURE VI. PROCESS OF SIMULATION PROGRAMMING.

\section{CASE STUdy}

The CMM simulation system in Fig (a) was developed by MFC. An Xml file named IP Xml which was generated by CAIP system would be imported. After importing this file, all of the paths in this file would be traversed. By choosing the corresponding path, the inspection items would be listed on the right. The name and path of the program should be given by the inspector. Then the parameters of the probe should be set. Eventually, the program would be generated.

After opening VERICUT and configuring the environment of the virtual CMM system, the program would be loaded. Then press start button and the simulation program would be executed.
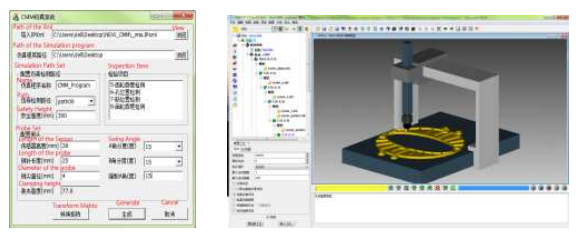

FIGURE VII. CASE STUDY.

\section{CONCLUSION}

In this paper, a new train of thought in inspection simulation was raised on the platform of VERICUT, which is one of the most common CNC simulation software. A CMM simulation system was established, where the Xml file generated by CAIP system was analysed and a CMM simulation program which could be loaded by VERICUT was generated. By building a virtual CMM system in VERICUT according to the actual measurement environment, the inspecting process could be simulated accurately by running the program.

\section{REFERENCES}

[1] Cen Rong. Research on Detection Path Planning and Simulation Based on Three-Dimensional Model [D]. Beihang University, Master's Thesis, 2013.
[2] Zhang Jiang. Research and System Development on Three-Dimensional Inspection Planning for Typical Aircraft Structural Parts [D]. Beihang University, Master's Thesis, 2009.

[3] Zheng Weilian. Study on Inspection Data Analysis and Visualization Technology based on Three-Dimensional Model.

[4] Tang X. Q., Davies B. J.. Integration of Inspection Planning System and CMM via DMIS[J]. The International Journal of Advanced Manufacturing Technology, 1995, 10(6): 422-426.

[5] Hwang C. Y., Tsai C. Y., Chang C. A. . Efficient inspection planning for coordinate measuring machines[J]. Int J Adv Manuf Technol, 2004, 23: $732-742$. 